\title{
Lifespan changes in global and selective stopping and performance adjustments
}

\author{
Maria C. van de Laar $^{1}{ }^{*}$, Wery P. M. van den Wildenberg ${ }^{1}$, Geert J. M. van Boxtel ${ }^{2}$ and \\ Maurits W. van der Molen ${ }^{3}$ \\ ${ }^{1}$ Amsterdam Center for the Study of Adaptive Control in Brain and Behavior, Department of Psychology, University of Amsterdam, Amsterdam, Netherlands \\ ${ }^{2}$ Department of Psychology, Tilburg University, Tilburg, Netherlands \\ ${ }^{3}$ Department of Psychology, University of Amsterdam, Amsterdam, Netherlands
}

\section{Edited by:}

Shulan Hsieh, National Cheng Kung

University, Taiwan

\section{Reviewed by:}

Cindy Lustig, University of Michigan, USA

Antonino Vallesi, Scuola Internazionale Superiore di Studi Avanzati, Italy

\section{*Correspondence:}

Maria C. van de Laar, Department of Psychology, University of Amsterdam, Weesperplein 4, 1018 XA Amsterdam, Netherlands.

e-mail:m.c.vandelaar@uva.nl
This study examined stopping and performance adjustments in four age groups ( $M$ ages: 8 , 12,21 , and 76 years). All participants performed on three tasks, a standard two-choice task and the same task in which stop-signal trials were inserted requiring either the suppression of the response activated by the choice stimulus (global stop task) or the suppression of the response when one stop-signal was presented but not when the other stop-signal occurred (selective stop task). The results showed that global stopping was faster than selective stopping in all age groups. Global stopping matured more rapidly than selective stopping. The developmental gain in stopping was considerably more pronounced compared to the loss observed during senescence. All age groups slowed the response on trials without a stop-signal in the stop task compared to trials in the choice task, the elderly in particular. In addition, all age groups slowed on trials following stop-signal trials, except the elderly who did not slow following successful inhibits. By contrast, the slowing following failed inhibits was disproportionally larger in the elderly compared to young adults. Finally, sequential effects did not alter the pattern of performance adjustments. The results were interpreted in terms of developmental change in the balance between proactive and reactive control.

Keywords: stop-signal paradigm, development, cognitive aging, lifespan, cognitive control

\section{INTRODUCTION}

The ability to dynamically adjust to the changing environment is critical for survival. One important component of this ability is to refrain from responding when a readied response is no longer required. In the laboratory, the ability to withhold motor responses has been examined frequently by using the stop-signal paradigm (Logan and Cowan, 1984; Verbruggen and Logan, 2008). In this paradigm, participants are typically presented with a stimulus requiring either a speeded left- vs. right-hand response. On some trials, a stop-signal is presented, occasionally and unpredictably, just following the onset of the choice stimulus indicating that the response activated by the choice stimulus should be withheld. Assuming that the go process activated by the choice stimulus and the stop process activated by the stop-signal are involved in a race, won by whichever process finishes first, allows for an estimate of the duration of the stop process, or stop-signal reaction time (SSRT, Logan and Cowan, 1984). Most stop studies use a global stop-signal that requires participants to stop whatever response is activated by the choice stimulus. Global SSRT is typically around $200 \mathrm{~ms}$ (Logan, 1994; Verbruggen and Logan, 2008). Some stop studies used a selective stop-signal indicating that the response activated by the choice stimulus should be withheld whereas the other (invalid) stop-signal indicates that the readied response is to be executed. Selective SSRT is typically longer than global SSRT, about $30 \mathrm{~ms}$ (Bedard et al., 2002; van den Wildenberg and van der Molen, 2004a; van de Laar et al., 2010). The first aim of the current study is to examine developmental trends in global and selective stopping along the lifespan.

Developmental studies showed that global stopping is usually slower in children than adults (Williams et al., 1999; but see Oosterlaan and Sergeant, 1996; Band et al., 2000; Johnstone et al., 2007). With advancing age the speed of global stopping seems to decline during senescence (Kramer et al., 1994; May and Hasher, 1998; Ridderinkhof et al., 1999; Rush et al., 2006; Gamboz et al., 2009) but the age-related decline in global stopping speed has been observed to be considerably less than the developmental gain during childhood. Thus, a lifespan analysis of global stopping revealed that older children (11 years) stopped about $50 \mathrm{~ms}$ faster than young children ( 7.5 years). By contrast, the elderly (60-81 years) were only about $20 \mathrm{~ms}$ slower in global stopping compared to young adults (Williams et al., 1999). The age-related decline was even absent in a study reported by Kray et al. (2009). Larger declines have been observed by Kramer et al. (1994) and May and Hasher (1998) but in these studies the primary choice task was more difficult than in the Williams et al. (1999) study and, thus, the cognitive demands imposed by the primary choice might have reduced the resources available for stopping, in particular in the elderly (cf., Williams et al., 1999).

Although only a few studies investigated developmental change in selective stopping, the results that emerged from these studies are similar to the pattern observed for global stopping. The lifespan study of selective stopping reported by Bedard et al. (2002) 
showed that selective stopping was slower; $248 \mathrm{~ms}$ in young adults compared to $209 \mathrm{~ms}$ for global stopping as observed previously by Williams et al. (1999). Furthermore, it was observed that the speed of selective stopping improved until young adulthood indicating that selective stopping takes longer to mature than global stopping. Finally, the difference between young adults and the elderly was about $60 \mathrm{~ms}$ whereas it was only $21 \mathrm{~ms}$ for global stopping, as observed by Williams et al. (1999), indicating that selective stopping may impose a larger burden on the elderly compared to global stopping. We observed a similar developmental pattern in a direct comparison of global vs. selective stopping (van den Wildenberg and van der Molen, 2004a). That is, selective stopping was slower than global stopping, in young adults (i.e., 232 vs. $205 \mathrm{~ms}$ ), and selective stopping matured slower than global stopping.

The current study will provide a direct comparison between age-related change in global vs. selective stopping along the lifespan. Such a comparison is still lacking in the stop-signal literature and may reveal potentially interesting differences in the developmental trends associated with global vs. selective stopping. A particular focus of the current study was on the performance on invalid stop-signal trials of the selective stop task. Invalid stopsignal trials are trials on which a stop-signal is presented that should be ignored by the participant. In our previous studies we observed that the speed of responding on invalid stop-signal trials was considerably delayed compared to trials without a stop-signal (van den Wildenberg and van der Molen, 2004a,b; van de Laar et al., 2010). Thus the stop-signal is not ignored by the participants (or not ignored on a substantial proportion of the invalid trials). Previously, we have argued that the delay might be due to the initial inhibition of all responses and the subsequent re-activation of the appropriate response following the complete analysis of the information provided by the stop-signal (van de Laar et al., 2010). In this regard, the performance on these trials might provide valuable information regarding the strategy employed by the participant. Based on previous findings (Band et al., 2000), we predicted that the re-initiation strategy would be more prominent in children compared to young adults. The current study would be first in providing data regarding invalid stop-signal trial performance in the elderly.

The second aim of the current study is to examine performance adjustments related to the insertion of stop-signal trials in the choice reaction task (CRT). Several studies focused on performance adjustments in the stop-signal paradigm (e.g., Rieger and Gauggel, 1999; Verbruggen and Logan, 2008, 2009; Verbruggen et al., 2008; Aron, 2011; Bissett and Logan, 2011). Rieger and Gauggel (1999) observed that the speed of responding to the choice stimulus was delayed on trials following a stop-signal trial. The delay was somewhat larger following a failed inhibit compared to following a successful inhibit and larger for trials repeating the choice stimulus compared to trials with alternating stimuli. The delay in responding following successful inhibits has been attributed to repetition priming (Verbruggen et al., 2008) and the added delay after failed inhibits has been interpreted in terms of trial adjustments or reactive control (Bissett and Logan, 2011).

Another type of control that might be exercised in the stop-signal task is referred to as proactive control (Verbruggen and Logan, 2009; see for a review Aron, 2011). In stop-signal experiments, participants are typically instructed not to wait for a stop-signal to occur. In spite of these instructions and despite experimental manipulations used to counter waiting strategies (e.g., the use of a tracking algorithm, see below) participants tend to slow their response when stop-signal trials are inserted in the CRT (Logan and Burkell, 1986; Ramautar et al., 2004; van de Laar et al., 2010). The slowing of choice reactions when stop-signal trials are inserted is interpreted in terms of a proactive change in response strategy aimed at increasing the success of stopping at the cost of the speed of choice reactions (e.g., Verbruggen and Logan, 2009).

Based on the performance data currently available we were led to predict that young adults would slow when stop-signal trials are inserted in the choice task (a manifestation of proactive control) and following stop-signal trials (indicating repetition priming when the choice stimulus is repeated and reactive control for alternating stimuli). Assuming that both children (e.g., Huizinga and van der Molen, 2010; Somerville and Casey, 2010) and the elderly (e.g., West and Schwarb, 2006; Mayda et al., 2011) are less efficient in implementing control procedures, we assumed that the response slowing associated with proactive and reactive control would be more pronounced in these age groups compared to young adults. Finally, it was predicted that the slowing associated with repetition priming would be more pronounced for children than adults based on recent findings indicating that the effects of repetition priming decrease when children are growing older (e.g., Crone et al., 2004; Smulders et al., 2005) and less pronounced or even absent for the elderly based on the repetition priming literature showing that repetition priming effects are basically age-invariant during senescence (e.g., Fleischman, 2007; Bergerbest et al., 2009, for reviews).

\section{MATERIALS AND METHODS PARTICIPANTS}

Four age groups participated in the study. There were two groups of children (a young child group of 8-years-old and an older child group of 12-years-old) and two groups of adults (young adults of 21-years-old and elderly adults of 76-years-old). Participant characteristics are presented in Table 1. Children were recruited from local elementary schools and selected with the help of their teachers. Informed consent was obtained from their primary caregivers. The children received a small present for participation. Young adults were university students who received course credits for participation. Healthy elderly participants were recruited from the metropolitan area. They received 40 euro for their participation. Informed consent was obtained from the adult participants.

Table 1 | Participant characteristics.

\begin{tabular}{llccl}
\hline Age group & Number & Age (M, SD) & Gender (f/m) & $\begin{array}{l}\text { Raven } \\
\text { percentile }\end{array}$ \\
\hline Young children & 20 & $7.6(0.59)$ & $10 / 10$ & $90(13.4)$ \\
Older children & 20 & $11.9(1.23)$ & $7 / 13$ & $79(17.5)$ \\
Young adults & 17 & $20.8(3.30)$ & $9 / 8$ & $88(3.6)$ \\
Elderly adults & 19 & $75.6(5.68)$ & $8 / 11$ & $92(4.9)$
\end{tabular}


All participants had normal or corrected-to-normal vision. The study was approved by the local Ethics Committee.

Chi-square analyses indicated that gender distribution did not differ between groups, $\chi^{2}(3)=3.36, p=0.34$. To compare estimates of general levels of intelligence between age groups, the standard progressive matrices (SPM; Raven et al., 1985) was administered to children and the elderly, whereas young adults completed the Advanced Progressive Matrices (APM, Raven et al., 1993). Mean percentile scores differed significantly across age groups, $F(3,70)=4.53, p<0.006$. As can be seen in Table 1 , the older children had a lower percentile score compared to the other age groups $(p s<0.03)$. Additional analyses verified that significant outcomes survived when using Raven percentile scores as covariate.

\section{MATERIALS AND STIMULI}

Participants were seated in a comfortable chair facing a computer screen at a distance of about $1.5 \mathrm{~m}$. Each trial started with a white fixation cross $(3 \times 3 \mathrm{~mm})$ appearing in the center of the screen for $500 \mathrm{~ms}$ followed by the go-signal. The go-signal consisted of a white left- or a right-pointing arrow $(2.2 \mathrm{~cm} \times 1.8 \mathrm{~cm})$ presented centrally for $1000 \mathrm{~ms}$ against a dark-gray background subtending a visual angle of $1.9^{\circ}$. The inter-trial interval varied randomly between 1750 and $2250 \mathrm{~ms}$, in steps of $50 \mathrm{~ms}$. During this interval, the fixation cross was presented. Motor responses were collected until the offset of the arrow stimulus. On 35\% of the trials the color of the white arrow changed to pink or blue, indicating a stop-signal. The stop-signal delay (SSD; i.e., the interval between the onset of the go-signal and the stop-signal) of the first stop trial in the practice block was set at $225 \mathrm{~ms}$ and was dynamically adjusted after valid stop trials throughout the experiment as a function of the stopping performance of the participant. Upon successful stopping, SSD on the subsequent stop trial increased with $25 \mathrm{~ms}$, whereas a failure to inhibit decreased SSD on the next stop trial by $25 \mathrm{~ms}$. This tracking algorithm (Levitt, 1971) was set to ensure $50 \%$ failed inhibits, which yields accurate estimates of stop-signal RT (Band et al., 2003).

Responses were collected by pressing a left or right zerodisplacement force sensor (Honeywell, model FS03) with the leftor right thumb to the direction of the arrow stimulus. Thumbs rested on force sensors that were individually adjusted according to the arm length of the participant. A continue sample frequency was applied $(>1000 \mathrm{~Hz})$ and the proportional voltage signal was A/D converted online (Keithley, model KPCI-1802 using DrvrLINX4). The value of $15 \%$ of the maximum force served as individual RT threshold which was determined prior to the experiment for each thumb separately (van Boxtel et al., 2001).

\section{EXPERIMENTAL TASKS}

The choice RT task CRT consisted of go-signals only, stop-signals were not included. Participants were instructed to press the left or right thumb to a white arrow pointing left or right, respectively. The CRT task consisted of one experimental block of 50 trials. All blocks contained equal numbers of right- and left-pointing arrows that were varied randomly within a block.

When performing the global stop-signal task (GST), participants were required to respond to go-signals according to the
CRT instruction described above. Trials without a stop-signal are referred to as go trials. On $35 \%$ of the trials, the stop-signal occurred instructing the participant to refrain from responding. The stop-signal consisted of either a blue or pink color change of the white arrow (counterbalanced across participants). Participants were informed about the tracking algorithm and it was explained that a "waiting" strategy would not increase the success of stopping. The GST consisted of four experimental blocks, each containing 100 trials.

In the selective stop-signal task (SST), participants were instructed to respond to go trials as described above, but in this task both stop-signals occurred. Participants were required to discriminate between stop-signals and to inhibit their response to one stop-signal (e.g., white-to-pink color change) but to ignore the other stop-signal (e.g., white-to-blue color change). Stop-signal colors were counterbalanced across participants. Stop trials requiring participants to stop were coined "valid" stop-signal trials and stop trials on which the stop-signal should be ignored were coined "invalid" stop-signal trials (e.g., van de Laar et al., 2010). Stopsignals occurred on $35 \%$ of the trials - half of the trials were valid and the other half of the trials were invalid. Participants performed eight experimental blocks of 100 trials.

\section{PROCEDURE}

The participants performed on all three experimental tasks - CRT, GST, and SST. The tasks were administered during two separate sessions on different days that were scheduled within 5 days. Each session started with the CRT task followed by one of the stop tasks. The order of the two stop tasks was counterbalanced across participants. The duration of an experimental trial block was approximately $5 \mathrm{~min}$ and each block was followed by performance feedback. Each task started with one practice block followed by the experimental blocks. A block started with five warm-up trials that were discarded from analyses. Short breaks were given between blocks and a longer rest was given between tasks.

\section{DATA ANALYSES}

Data of six young children (CRT data from five children and GST data from one child), an older child (CRT data), and one elderly participant (SST data) were discarded from analyses, because of unreliable recordings of force pressure. Repeated measures analyses of variance (ANOVA) were conducted on latency measures (median RT) and error proportions (errors and omissions). Multiple comparisons were used to confirm effects. Degrees of freedom and $p$-values were adjusted using Greenhouse-Geisser corrections. Since error proportions are not normally distributed, tests were performed on square rooted error values.

The integration method based on the horse-race model was used to obtain estimates of stopping latencies (the stop-signal RT or SSRT, Logan and Cowan, 1984). Using this model, the finish of the stop process can be estimated from the go RT distribution. The left side of the distribution of the RTs on go trials represents fast responses that escape inhibition whereas the right side represents slow responses that will be inhibited. If a given participant actually failed to inhibit on $n \%$ of the stop trials, the finishing time of the stop process will approximately be equal to the $n$th percentile of the go RT distribution. The mean SSD is then subtracted from the 
$n$th percentile of the go RT distribution, resulting in an estimate of SSRT (see Figure 1).

Performance adjustments following a stop trial were examined by comparing trial duplets (go-go vs. successful stop-go vs. failed stop-go vs. invalid stop-go). Within duplets, repetitions vs. alternations were distinguished. A repetition duplet consisted of a trial in which the go stimulus indicated the same response as the one on the immediately preceding trial, whereas an alternation duplet consisted of a trial on which the go stimulus indicated the opposite response.

Significant interaction effects including Age Group were analyzed further by transforming RTs to the natural logarithm to reduce the influence of differences between age groups in baseline performance (e.g., Meiran, 1996; Huizinga and van der Molen, 2010). Thus, interactions resulting from ANOVA on the transformed data indicate a disproportional difference in RT between age groups in one condition relative to another.

\section{RESULTS}

The results will be presented in three major sections. In the first section, we will examine age-related trends in the speed of responding on go trials and will ask whether these trends differ between tasks (CRT, GST, and SST). A particular question that

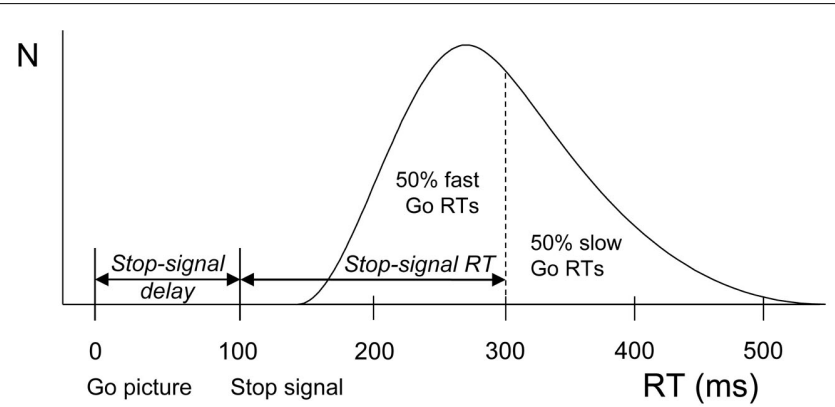

FIGURE 1 | Schematic representation of the race model. A distribution of reaction times (RTs) on go trials (trials without a stop-signal) is shown beneath the curve. These values can be seen as finishing times of the go process. In stop trials, a stop-signal was shown after the go-signal at a particular stop-signal delay. The finishing time of the stop process bisects the go-signal RT distribution. The left part consists of go-signal RTs fast enough to escape inhibition (i.e., 50\%). The right part (50\%) represents slow go-signal RTs that will be inhibited because the stop process finished before. Stop-signal RT (200 ms) is estimated by subtracting average stop-signal delay $(100 \mathrm{~ms})$ from the RT that marks the bisection point (300 ms) will be addressed refers to age-related changes in the speed of responding when stop-signals are inserted into the task. Based on previous studies, we anticipated that, in spite of the tracking algorithm, participants would delay their speed of responding on go trials when they might encounter stop-signals (Verbruggen and Logan, 2009; Aron, 2011).

In the second section, we focus on age-related changes in the speed of stopping and we will ask whether trends differ between global vs. selective stopping. A second question that will be addressed in this section is on the speed of responding when stopping fails (i.e., failed inhibits). Based on the horse-race model underlying the stop-signal paradigm (Logan and Cowan, 1984), it would be predicted that the failed inhibits are faster than responses on go trials. We anticipated obtaining this pattern for each of the age groups. Finally, we will focus on the speed of responding on invalid stop-signal trials. In previous studies, we observed that the speed of responding on invalid stop-signal trials was considerably slower than on go trials (van den Wildenberg and van der Molen, 2004a; van de Laar et al., 2010). Age-related differences in the slowing on invalid stop-signal trials may contribute to our understanding of how stopping is realized in each of the age groups participating in the current study. In the third section, we will examine performance adjustments following stop-signal trials. First, we asked whether age groups differed in the speed of responding following a successful inhibit relative to the speed on a go trial following another go trial. Second, we asked whether the age groups differed in the speed of responding following a failed inhibit. Third, we examined age-related changes in the speed of responding following an invalid stop-signal trial. For all analyses we examined whether performance adjustments differed between repetition vs. alternation trials. Obviously, it would be of considerable interest to assess whether age-related patterns in performance adjustment are similar across trial type suggesting a single underlying mechanism.

\section{PERFORMANCE ON GO TRIALS}

Median RTs and error percentages on go trials are presented in Table 2 for each task (CRT, GST, and SST) and age group. The typical lifespan pattern is observed for the RTs of each task - the speed of responding increases into adulthood and slows down in the elderly (see Figure 2). It can be seen that, in spite of the instructions and the dynamic tracking of SSD, all age groups slowed their speed of responding when the task included stop-signals. For all age groups, the slowing seems more pronounced on the GST compared to the SST. These visual impressions were verified

Table 2 | Median RTs and error percentages associated with go trials of the choice reaction task (CRT), global stop-signal task (GST), and the selective stop-signal task (SST) in each age group (SD between parentheses).

\begin{tabular}{|c|c|c|c|c|c|c|c|c|c|}
\hline $\begin{array}{l}\text { Task } \\
\text { Age group }\end{array}$ & \multicolumn{3}{|c|}{ CRT } & \multicolumn{3}{|c|}{ GST } & \multicolumn{3}{|c|}{ SST } \\
\hline Older children & 373 (45.3) & $8.0(5.7)$ & $0.1(0.5)$ & $462(56.8)$ & $3.2(2.5)$ & $1.2(2.5)$ & 426 (45.6) & $4.2(2.9)$ & $0.5(0.9)$ \\
\hline Young adults & $296(22.2)$ & $2.7(3.0)$ & $0.1(0.5)$ & 342 (25.9) & $1.3(1.7)$ & $0.2(0.4)$ & 326 (28.6) & $1.5(1.9)$ & $0.0(0.1)$ \\
\hline Elderly adults & $435(61.7)$ & $3.2(2.8)$ & $0.1(0.5)$ & $631(153.7)$ & $1.5(1.7)$ & $0.6(1.2)$ & 601 (137.8) & $0.9(1.2)$ & $1.0(2.3)$ \\
\hline
\end{tabular}


by submitting median RTs to ANOVA with Task (3), as a within Ss factor, and Age Group as a between Ss factor. The ANOVA yielded main effects of Task, $F(2,128)=82.66, p<0.001$, and Age Group, $F(3,64)=49.35, p<0.001$, and their interaction was significant also, $F(6,128)=8.47, p<0.001$. Subsequent analyses indicated that all age groups showed a delay in responding associated with the insertion of stop-signals ( $p s<0.001)$. The delay was more pronounced for the GST compared to the SST in the child groups and young adults ( $p s<0.002$ ), but for the elderly adults the delay did not differ between tasks $(p=0.44)$. Log transformed analyses indicated that the delay was disproportionally larger in the elderly adults compared to the other age groups on both stop-signal tasks ( $p s<0.007$ ). The delay was disproportionally larger for the young children compared to young adults on the GST $(p<0.02)$, and marginally larger for the SST $(p=0.06)$.

A similar analysis was performed on the error percentages. Analyses yielded a main effect of Task, $F(2,128)=20.03, p<0.001$, and Age Group, $F(3,64)=17.51, p<0.001$, but their interaction was not significant, $F(6,128)=1.54, p=0.19$. Subsequent analysis revealed higher error rates on the CRT compared to the stopping tasks $(p s<0.001)$. Young children committed more errors compared to older children $(p=0.04)$, and older children made more errors than the two adult groups $(p s<0.001)$. Error rates did not differ between the two adult groups $(p=0.91)$.

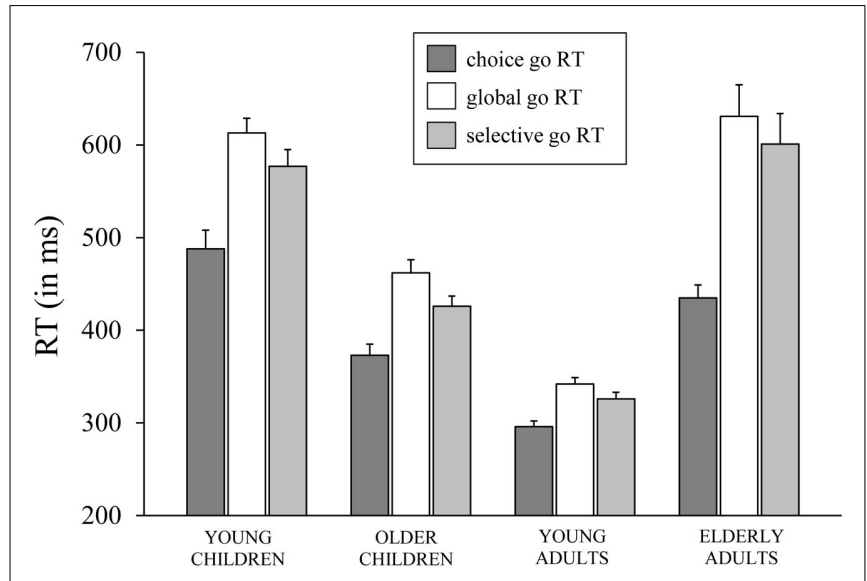

FIGURE 2 |The speed of responding (in ms) on go trials (go RT) of the choice reaction task, the global stop-signal task, and the selective stop-signal task for each of the four age groups (8-, 12-, 21-, and 76-years-old).
Analyses on omission errors (i.e., errors associated with a failure to respond to the go-signal) yielded a significant main effect of Task, $F(2,128)=8.72, p<0.001$, and Age Group, $F(3$, $64)=15.64, p<0.001$, but their interaction was not significant, $F(6,128)=1.12, p=0.35$. Omission rate was higher on the stopping tasks compared to the CRT $(p s<0.002)$. Young children committed more omissions than the other age groups $(p s<0.001)$.

\section{PERFORMANCE ON STOP-SIGNAL TRIALS}

Stop-signal reaction time, SSD, and the percentage of successful inhibits are presented in Table $\mathbf{3}$ for each age group and both stop-signal tasks. It can be seen that the SSRTs are relatively short in both tasks. Typically, young adults' global SSRT to auditory stop-signals is about $200 \mathrm{~ms}$ (Logan, 1994; Verbruggen and Logan, 2008), and SSRT to visual stop-signals is longer compared to auditory ones (van der Schoot et al., 2005). The current SSRT to visual stop-signals, however, is only $181 \mathrm{~ms}$ in young adults. In a previous study, using a similar GST, adult SSRT was $210 \mathrm{~ms}$ (van de Laar et al., 2010). Most likely, the relatively short SSRTs in the present study are due to the specifics of the response device (see also van Boxtel et al., 2001). Force transducers were used in the current study whereas a computer keyboard was used in the study reported by van de Laar et al. (2010). Typically, computer keyboards involve a considerable delay in transmission time (e.g., Li et al., 2010).

The Task $\times$ Age Group ANOVA on SSRT yielded significant main effects of Task, $F(1,70)=138.28, p<0.001$, and Age Group, $F(3,70)=61.6, p<0.001$, which were included in a significant Task $\times$ Age Group interaction, $F(3,70)=15.71, p<0.001$, which is plotted in Figure 3. Subsequent analyses for each task, separately, yielded a significant effect of Age Group for the GST, $F(3,71)=40.98, p<0.001$. Analysis on the log transformed data showed that young children stopped slowest and young adults stopped fastest ( $p s<0.009$ ), with older children and elderly adults having intermediate stopping times $(p=0.67)$. The effect of Age Group was significant also for the SST, $F(3,71)=57.18$, $p<0.001$. Again, analysis on log transformed SSRTs indicated that young children stopped slowest and young adults stopped fastest $(p s<0.03)$. But now, older children stopped disproportionally faster than elderly adults $(p<0.001)$. Finally, selective stopping was slower than global stopping in all age groups $(p s<0.001)$. The analysis on log transformed data revealed that stopping speed on the SST relative to GST was disproportionally slower in the older and young child groups (respectively 1.4 and 1.3 times,

Table 3 | Mean stop-signal reaction time (SSRT), stop-signal delay (SSD), and percentage inhibits associated with the global stop-signal task (GST) and the selective stop-signal task (SST) in each age group (SD between parentheses).

\begin{tabular}{|c|c|c|c|c|c|c|}
\hline $\begin{array}{l}\text { Task } \\
\text { Age group }\end{array}$ & \multicolumn{3}{|c|}{ GST } & \multicolumn{3}{|c|}{ SST } \\
\hline Older children & 203 (29.6) & $256(61.9)$ & $50.0(1.3)$ & $277(47.2)$ & 149 (55.3) & $49.0(2.9)$ \\
\hline Young adults & $181(17.4)$ & $159(27.2)$ & $50.6(0.9)$ & $203(26.8)$ & $124(32.9)$ & $49.6(1.7)$ \\
\hline Elderly adults & $206(23.5)$ & 416 (154.4) & $52.0(2.6)$ & $224(23.7)$ & 368 (132.2) & $52.1(2.7)$ \\
\hline
\end{tabular}


$p=0.78)$ compared to the two adult groups (1.1 times, $p=0.72$; ps $<0.001)$.

Finally, as can be seen in Table 3, the percentage of successful inhibits was close to $50 \%$ for all groups on both stopping tasks. This indicates that the tracking algorithm, which was targeted at 50\% successful inhibits, worked quite well. The analysis done on the square rooted proportions showed that the proportion of successful inhibits was somewhat higher on the GST compared to the SST, $F(1,70)=5.27, p=0.03$. Stopping success was somewhat higher in the elderly compared to the other age groups $(p s<0.006)$.

Table 4 presents the RTs associated with failed inhibit responses for both stopping tasks and the RTs associated with invalid stopsignal trials of the SST together with the percentage of omissions on this task (i.e., an invalid stop-signal was presented but the participant failed to respond). The age-related changes in the speed of failed inhibits and responses on invalid stop-signal trials are plotted in Figure 4.

First, the Task $\times$ Age Group ANOVA revealed that failed inhibit RTs were shorter than go RTs on the GST for all age groups, $F s>64.43, p s<0.001$, as predicted by the horse-race model underlying the analysis of stopping speed (e.g., Logan and Cowan,

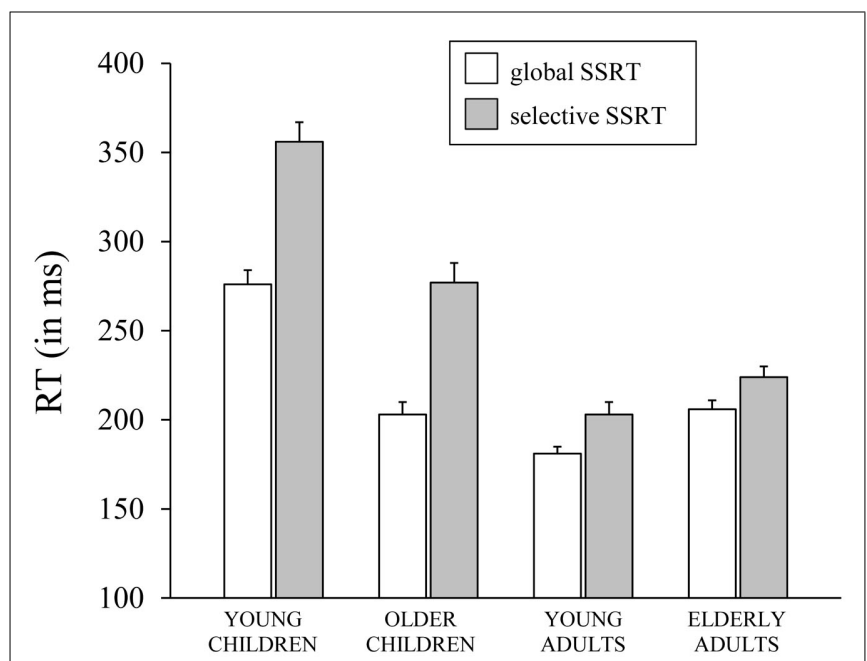

FIGURE 3 |The speed of stopping (in ms, SSRT) on the global stop-signal task and the selective stop-signal task for each of the four age groups (8-, 12-, 21-, and 76-years-old).

Table 4 | Median failed inhibit reaction time (FIRT) for the global stop task (GST) and the selective stop task (SST).

\begin{tabular}{lllll}
\hline \multirow{2}{*}{ Task } & GST & \multicolumn{3}{c}{ SST } \\
\cline { 3 - 5 } Age group & FIRT (ms) & FIRT (ms) & IRT (ms) & Omissions (\%) \\
\hline Young children & $519(53.0)$ & $517(56.7)$ & $648(77.4)$ & $3.6(3.5)$ \\
Older children & $403(40.1)$ & $400(41.4)$ & $503(78.0)$ & $1.1(1.7)$ \\
Young adults & $316(21.3)$ & $320(30.7)$ & $394(37.0)$ & $0.6(1.1)$ \\
Elderly adults & $554(134.4)$ & $532(110.3)$ & $721(155.0)$ & $2.6(3.5)$
\end{tabular}

Median invalid reaction time (IRT) and proportion of omissions for the SST in each age group (SD between parentheses).
1984). This observation is consistent with the notion that failed inhibits are fast responses escaping inhibition. For the SST, failed inhibit RTs were shorter than go RTs for the two child groups and elderly adults, $F s>48.69$, ps $<0.001$, but not for young adults, $F(1,16)=2.66, p=0.12$. Failed inhibit RTs did not differ between stop tasks, $F<1$, but there was a substantial difference across age groups, $F(3,70)=45.85, p<0.001$. Log transformed analysis indicated that failed inhibit RT was shortest in young adults compared to all three other age groups ( $p s<0.001)$. Failed inhibit RT was shorter in older children relative to young children and the elderly $(p s<0.001)$. Failed inhibit RT did not differ between young children and the elderly $(p=0.58)$.

Secondly, in Table 4 it can be seen that failed inhibit RTs are basically similar across the two stopping tasks suggesting that the impact of stop-signal processing on the response process must have been minimal or even absent on these trials. In contrast, the responses on invalid stop trials of the SST are very slow; much slower than the speed of responding on (non-signal) go trials. The Task $\times$ Age Group analysis revealed that invalid RTs were longer compared to go RTs in all age groups, $F(3,71)=4.76, p=0.004$. The analysis on log transformed data indicated that the slowing on invalid stop trials relative to go trials was disproportionally larger in elderly adults compared to the other age groups ( $p s<0.009$ ).

Finally, performance on invalid stop-signal trials was relatively accurate. The proportion of omissions remained below $5 \%$ for each age group. Statistical analysis yielded a significant Age Group effect on the proportion of omissions, $F(3,71)=6.2, p<0.001$. Elderly adults failed to respond somewhat more frequently on invalid stop trials than young children and young children failed to respond somewhat more frequently than older children and young adults $(p s<0.01)$.

\section{PERFORMANCE ADJUSTMENTS}

\section{Go trials following go trials}

To assess sequential effects on go RTs following go trials, we performed an ANOVA including Task (3), and Sequence (2) as within Ss factors and Age Group (4) as between Ss factor (see Table 5). The analysis yielded a significant interaction between Age Group and

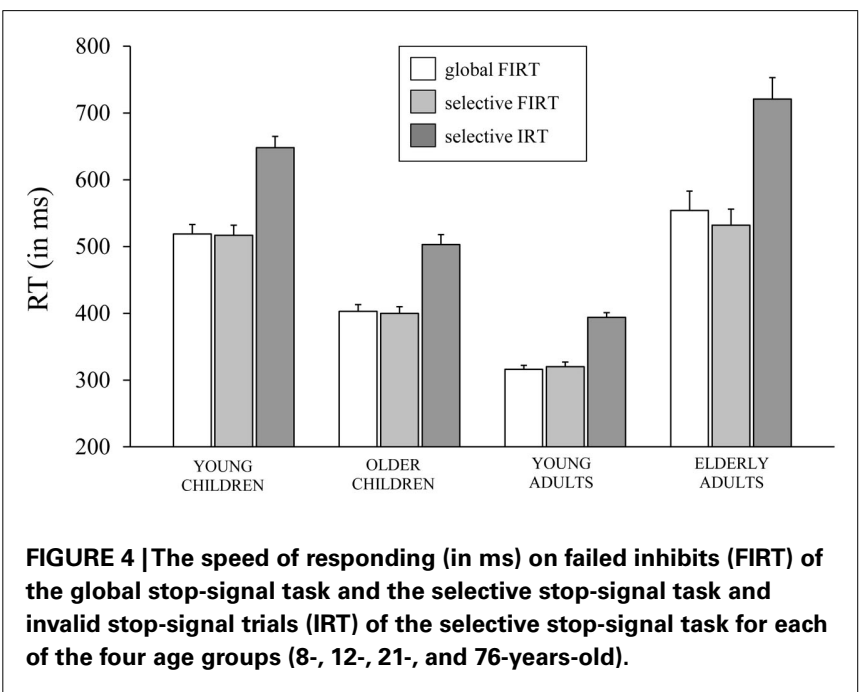


Sequence, $F(3,64)=8.11, p<0.001$. Sequence did not interact with Task, $F<1$. Follow-up analysis indicated that the speed of responding on repetitions was similar to alternations in young adults ( 315 vs. $319 \mathrm{~ms}, p=0.09$ ) whereas the three other age groups showed the opposite pattern (564 vs. $526 \mathrm{~ms}$ for younger children, $p<0.001 ; 423$ vs. $409 \mathrm{~ms}$ for older children, $p<0.01$; and 561 vs. $542 \mathrm{~ms}, p<0.04$, for elderly adults). Subsequent analysis on log transformed data indicated that the relative alternationrepetition difference was larger in younger children and smaller in young adults relative to the two other age groups $(p s<0.03)$. Older children did not differ from the elderly adults in this regard $(p=0.62)$.

\section{Go trials following stop trials}

The Task (2; GST and SST) $\times$ Duplet (3; go-go, successful inhibitgo, and failed inhibit-go) $\times$ Age Group (4) ANOVA yielded a significant main effect of Duplet, $F(2,142)=17.73, p<0.001$, which was included in a significant interaction with Age Group, $F(6,140)=3.49, p=0.003$. A significant main effect of Task was found, $F(1,70)=11.48, p=0.001$; the overall delay was larger on the GST compared to the SST. Neither Age Group nor Duplet interacted with Task, Fs $<2.12$. The data are presented in Table 6 and age-related trends are plotted in Figure 5.

The delay in responding following successful inhibits, relative to the speed of responding to go trials following non-signal (go) trials, was significant for the two child groups and young adults ( $p s<0.004)$, but not for elderly adults $(p=0.97)$. The analysis on the log transformed data indicated that the delay did not discriminate disproportionally between the child groups and young adults ( $p s>0.43$ ), but the delay was disproportionally longer for the child groups and young adults compared to elderly adults $(p s<0.02)$.
The delay of responding following failed inhibits compared to go trials was significant for all age groups $(p s<0.01)$. The analysis on the log transformed data failed to reveal disproportional differences between age groups in this regard $(p=0.50)$. The delay following a failed inhibit was similar to the delay following a successful inhibit for young children and young adults $(p s>0.11)$. Failed inhibit delay was larger than successful inhibit delay for elderly $(p=0.02)$, whereas the opposite pattern was observed for older children $(p=0.02)$. The analysis on log transformed data revealed that elderly adults delayed the go response following a failed inhibit compared to following a successful inhibit disproportionally more than the other age groups $(p s<0.02)$ and young children somewhat more than older children $(p=0.07)$. Overall, it appears that the delay following stop trials is dependent on whether the response on the immediately preceding trial was successfully stopped or not.

Performance adjustments following stop-signal trials may depend on trial-by-trial stimulus sequence (e.g., Verbruggen et al., 2008). The following analysis was conducted to assess the effect of repetition or alternation stimulus sequence on the performance adjustments following successful and failed inhibited trials. ANOVA was performed on Task (2), Duplet (2; successful inhibitgo, and failed inhibit-go), Sequence $(2) \times$ Age Group (4). The data are presented in Table 7. This analysis revealed a significant Sequence effect, $F(1,70)=91.93, p<0.001$. The speed of responding on repetitions $(528 \mathrm{~ms})$ was longer than on alternations (497 ms). Neither Sequence nor Task did interact with Duplet, $F<1$. There was a significant interaction between Sequence and Age Group, $F(3,70)=7.89, p<0.001$. Subsequent analysis on log transformed data indicated that the relative alternation-repetition difference was larger in younger children compared to older

Table 5 | Median RT (in milliseconds) for repetition and alternation on go trials following go trials of the choice reaction task (CRT), the global stop-signal task (GST), and the selective stop-signal task (SST) in each age group (SD between parentheses).

\begin{tabular}{|c|c|c|c|c|c|c|}
\hline \multirow{2}{*}{$\begin{array}{l}\text { Task } \\
\text { Age group }\end{array}$} & \multicolumn{2}{|c|}{ CRT } & \multicolumn{2}{|c|}{ GST } & \multicolumn{2}{|c|}{ SST } \\
\hline & Repetition & Alternation & Repetition & Alternation & Repetition & Alternation \\
\hline Young children & 517 (76.8) & 466 (71.0) & 619 (53.3) & $583(63.0)$ & 587 (79.8) & 559 (61.9) \\
\hline Older children & 383 (46.2) & 365 (52.7) & 458 (59.5) & $445(49.4)$ & $428(49.4)$ & 417 (43.2) \\
\hline Young adults & $297(25.7)$ & $296(22.0)$ & $330(24.7)$ & $337(25.7)$ & $319(28.3)$ & $325(29.6)$ \\
\hline Elderly adults & 445 (64.5) & $424(61.0)$ & $638(165.4)$ & $621(152.2)$ & 606 (168.3) & 591 (127.8) \\
\hline
\end{tabular}

Table 6 | Performance adjustments on the global stop-signal task (GST) and the selective stop-signal task (SST) for four age groups.

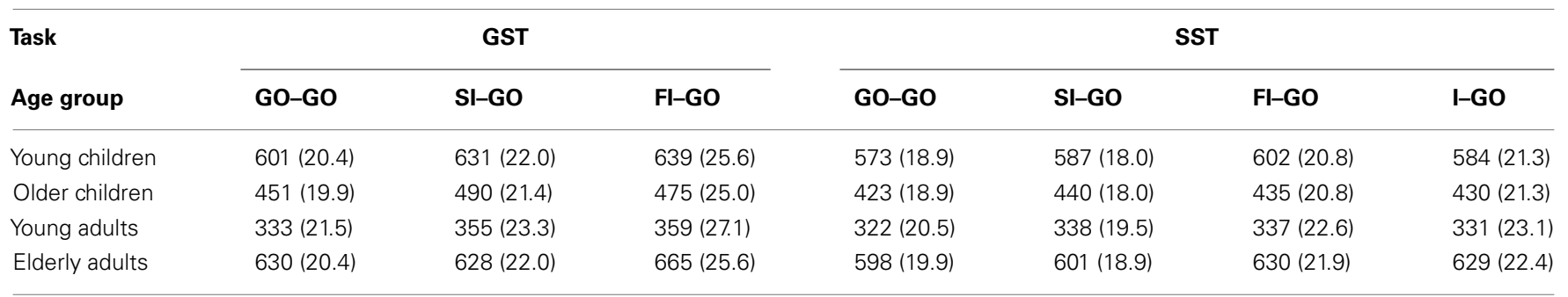

Median RTs (in milliseconds) are presented associated with go trials following a go trial (GO-GO), go trials following a successful inhibit (SI-GO), go trials following a failed inhibit (FI-GO) and, for the SST, go trials following an invalid stop-signal trial (I-GO; SD between parentheses). 


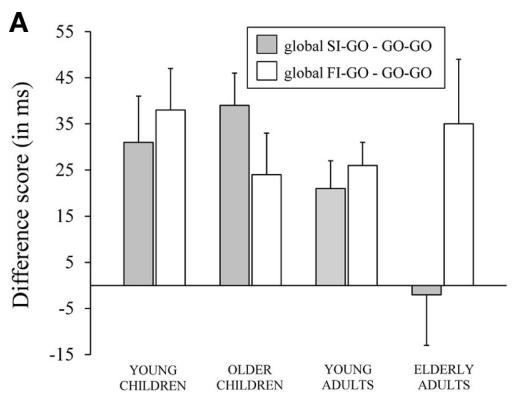

FIGURE 5 |The difference score between the speed of responding (in $\mathrm{ms}$ ) on go trials following successful inhibit trials (SI-GO) and on go trials following failed inhibit trials (FI-GO) relative to go trials following go trials (GO-GO) associated with the global stop-signal task (A) and speed of responding (in $\mathrm{ms}$ ) on go trials following

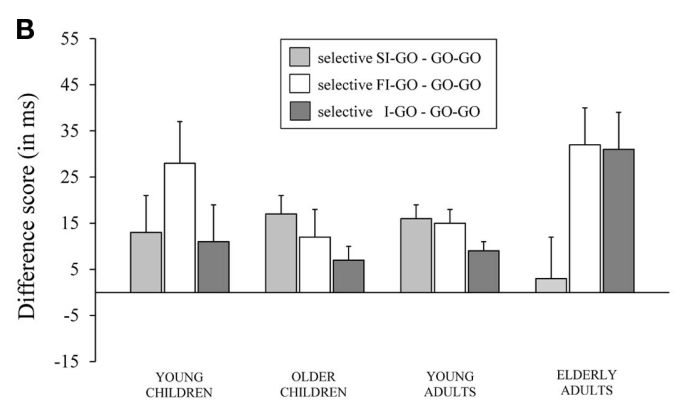

successful inhibit trials (SI-GO), on go trials following failed inhibit trials (FI-GO), and on go trials following invalid stop-signal trials (I-GO) relative to go trials following go trials (GO-GO) associated with the selective stop-signal task (B) for each of the four age groups (8-, 12-, 21-, and 76-years-old).

Table 7 | Median RT (in milliseconds) for repetition and alternation go trials following a successful inhibit (SI-GO), failed inhibit (FI-GO), and invalid stop (I-GO) of the global stop-signal task (GST) and the selective stop-signal task (SST) in each age group (SD between parentheses).

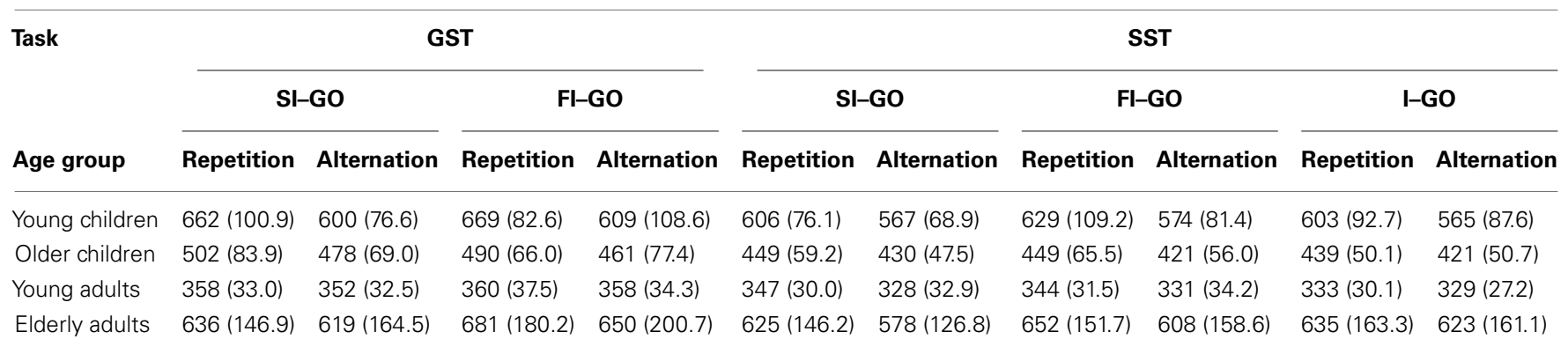

children and young adults ( $p$ s $<0.006)$, and tended to be larger compared to elderly adults $(p=0.06)$. The relative alternationrepetition difference was also larger in elderly adults compared to young adults $(p=0.02)$, but did not differ from the older children $(p=0.40)$. Older children did not differ from young adults either $(p=0.11)$.

A separate analysis was done on the SST task to test performance adjustments following invalid stop trials. The ANOVA Duplet (4; go-go, successful inhibit-go, failed inhibit-go, and invalid stop-go) $\times$ Age Group yielded a significant main effect of Duplet, $F(3,213)=12.34, p<0.001$, that interacted with Age Group, $F(9,213)=2.57, p=0.008$ (see Table 6). More specifically, the speed of performance adjustment following an invalid stop trial compared to following a go trial was larger in older children and in the two adult groups $(p s<0.02)$, but failed to reach significance in young children $(p=0.19)$. The log transformed analysis showed that elderly adults delayed the response following an invalid stop-signal trial relative to following a go trial disproportionally more compared to the other age groups $(p s<0.02)$. The delay following an invalid stop was disproportionally larger compared to following successful inhibits in the elderly adults compared to the other age groups $(p s<0.01)$. Finally, the delay following an invalid stop-signal trial did not significantly differ from those observed following failed inhibit trials in all age groups $(p s>0.07)$.
A final analysis was conducted to assess whether the performance adjustments following invalid stop-signal trials differed between repetitions vs. alternations. The Sequence (2) $\times$ Age Group (4) ANOVA yielded a significant Sequence, $F(1,71)=19.86, p<0.001$, that interacted with Age Group, $F(3$, $71)=3.12, p=0.03$. As can be seen in Table 7 , the delay following an invalid stop-signal trial was longer when the stimulus was repeated compared to when it was alternated for the two child groups ( $p s<0.005$ ), but not for the two adult groups ( $p s>0.16)$. The log transformed analysis showed that the relative alternation-repetition difference was disproportionally larger in young children compared to the two adults groups $(p s<0.04)$.

\section{DISCUSSION}

The current study aimed at examining lifespan changes in global and selective stopping and in post-stopping performance adjustments. With regard to the first aim, the results showed that global stopping was faster than selective stopping, which is typically interpreted in terms of the time consumed by additional processing (e.g., stimulus discrimination) in selective stopping (van den Wildenberg and van der Molen, 2004b; Verbruggen et al., 2005; van de Laar et al., 2010). It could be argued that a longer SSRT in the SST is due to the lower probability of valid stop-signals in this task relative to the global task (i.e., 17.5 vs. $35 \%$, respectively). A lower probability of stopping is typically associated with faster responses 
on go trials, as has been observed in both go/no-go paradigms (e.g., Luce, 1986) and in stop-signal paradigms (e.g., van den Wildenberg and van der Molen, 2003; Ramautar et al., 2004; van de Laar et al., 2010). Indeed, in the current study responses on go trials were faster on the selective stopping task compared to the global stopping task ( 483 vs. $513 \mathrm{~ms}$, respectively). The longer SSRT on the selective stop task is then explained by assuming that it is harder to stop because responses on go trials become more pre-potent when stop-signals are fewer. It should be noted, however, that in studies manipulating stop-signal probability, SSRT did not change (Ramautar et al., 2004; van de Laar et al., 2010) or was even shorter, not longer, when stop-signals were fewer (e.g., van den Wildenberg and van der Molen, 2003). Thus, at this point, the most likely interpretation of longer SSRTs on the SST is in terms of added signal discrimination demands.

Both global and selective SSRTs showed the typical U-shaped relation with advancing age albeit less pronounced than RTs on non-signal (go trials) or signal-respond trials (failed inhibits and invalid stop trials). The U-shaped relation between the processing speed and advancing age is a ubiquitous phenomenon (e.g., review in Cerella and Hale, 1994) that attracted a score of interpretations, including age-related changes in the setting of response thresholds (Starns and Ratcliff, 2010), information loss (Myerson et al., 1990), inhibitory control (Dempster, 1992), frontal lobe function (West, 1996), and neural noise (Kail, 1997).

A host of studies indicated that the ability to inhibit pre-potent responses improves rapidly when children are growing older (e.g., Ridderinkhof et al., 1999; Band et al., 2000; van den Wildenberg and van der Molen, 2004a) and starts to decline during senescence (e.g., Williams et al., 1999; Bedard et al., 2002). The current findings are basically consistent with this literature. More specifically, the current findings obtained for the GST showed that the speed of global stopping was slower for young children and faster for young adults compared to older children and elderly adults who did not differ in this regard. This pattern was similar for selective stopping with one exception; the speed of selective stopping did not discriminate between the young vs. elderly adults. It should be noted that the developmental gain in global stopping was larger during childhood (young vs. older children) than adolescence (older children vs. young adults). For selective stopping the developmental gain during childhood was basically similar to the gain during adolescence. This pattern is consistent with the findings reported previously by van den Wildenberg and van der Molen (2004a) and suggests that selective stopping matures slower than global stopping.

Based on a "last-in-first-out" hypothesis of lifespan changes in neurocognition (e.g., Davis et al., 2009), one would be led to predict that with advancing age selective stopping would be affected more than global stopping. The current findings, however, showed the opposite pattern. Global, but not selective, stopping discriminated significantly between young vs. elderly adults. Typically, global stopping is slower in the elderly compared to young adults (e.g., Williams et al., 1999; Rush et al., 2006; but see Kramer et al., 1994). The single study of selective stopping including elderly adults (Bedard et al., 2002) showed a pronounced difference between young vs. elderly adults in the speed of selective stopping; 248 vs. $329 \mathrm{~ms}$, respectively. In the current study, the difference between young vs. elderly adults was only 203 vs. $224 \mathrm{~ms}$, respectively. But the elderly adults differed from young adults in being disproportionally slow on invalid stop-signal trials (721 vs. $394 \mathrm{ms,}$ respectively).

One interpretation of the conspicuous absence of a sizable difference in the speed of selective stopping between young vs. elderly adults would be that on a proportion, if not all, of the trials, the elderly adults stopped all responses first and then decided to recruit the appropriate response after determining that inhibition is not required (e.g., Aron and Verbruggen, 2008; van de Laar et al., 2010). The deployment of a "stop-all" strategy in the elderly adults might be another instance of their inclination of preventing errors even when it slows them down doing so. It has been observed that a considerable portion of response slowing in the elderly is due to extra time consumed by response generation at the cortical level, as indicated by brain potentials (e.g., Kolev et al., 2005), the corticospinal level, as indicated by motor-evoked potentials (e.g., Fujiyama et al., 2011), and response activation, as indicated by movement kinematics (e.g., Trewartha et al., 2011). Moreover, it has been observed that the elderly show excessive response activation on no-go trials (e.g., Vallesi and Stuss, 2010; see also Vallesi, 2011; Vallesi et al., 2011). Thus, re-activation following the suppression of response-related over-recruited neural circuitry might be particularly time-consuming in the elderly (e.g., Vallesi, 2011; see also Vallesi and Stuss, 2010).

The second aim of the current study was to examine lifespan changes in post-stopping adjustment. Previous studies indicated that post-stopping adjustment is critically dependent on stimulusresponse repetitions and alternations across trials. That is, the speed of responding is delayed on post-stopping trials and the delay is largest when the stimulus is repeated (Verbruggen et al., 2008; Bissett and Logan, 2011). Verbruggen et al. (2008) argued that the goal to stop on successful inhibits replaces the go goal on the following go trial if the stimulus is repeated. In previous studies we examined age-related changes in trial-by-trial sequential effects (Melis et al., 2002; Smulders et al., 2005). This research showed that relatively long intervals between trials (i.e., >500 ms) are associated with a repetition benefit for young children (Smulders et al., 2005) and an alternation benefit for young and elderly adults (Melis et al., 2002). The former has been interpreted in terms of automatic facilitation or priming and the latter has been taken to be a manifestation of subjective expectancy (e.g., Gao et al., 2009). Our data deviate from previous findings in showing an alternation benefit for all age groups with the exception of young adults who failed to show significant sequential effects. One interpretation would be that the current discrepancy is due to the insertion of stop-signals into the trial series. This interpretation is unlikely since the alternation benefit associated with invalid stop trials was absent also in the two adult groups. Thus, an alternative interpretation assumes that subjective expectancies are relatively short-lived and dissipate rapidly so that their influence cannot be detected when inter-trial intervals are relatively long. Along those lines, it would be predicted that with increasing inter-trial intervals alternation benefits should disappear gradually in all age groups.

Most important, the current young adult results are consistent with previous reports showing a delay in the speed of responding on trials following successful or failed inhibits in GSTs (e.g., Rieger 
and Gauggel, 1999; Verbruggen et al., 2008). The current results add to this literature by showing that a similar pattern occurs on a SST. The present data revealed that responses following failed inhibits are not significantly slower than responses following successful inhibits. Similarly, responses following invalid stop-signal trials in the selective stop-signal trials were equally slow compared to responses following successful inhibits. In contrast to previous studies, the post-stop trial adjustments in the speed of responding were not influenced by sequential effects. Thus the current results cannot be interpreted easily in terms of repetition priming suggesting that the stop-signal on the previous successful inhibit trial automatically primes the stop process on the current trial (e.g., Verbruggen et al., 2008). Instead the current findings are compatible with several notions assuming that a stop-signal on the previous trial induces a switch to a more conservative response set resulting in a slowing of response execution. These notions assume that the conflict between responding and stopping induced by the stop-signal recruit control processes resulting in performance adjustments on the subsequent trial (e.g., Rieger and Gauggel, 1999; Botvinick et al., 2001) or that the infrequent stop-signal elicits an orienting response reducing the speed of responding on the subsequent trial (e.g., Notebaert et al., 2009). Along these lines it would be predicted that response slowing on trials following a stop-signal does not discriminate between successful vs. failed inhibit vs. invalid stop-signal trials.

To our surprise, the developmental findings did not differ from the results obtained in young adults. Response slowing following stop-signal trials on both the global and SSTs was somewhat more pronounced in young adults but not disproportionally so. Studies examining age-performance adjustments following stop-signal trials are scarce and focused on comparisons between clinical groups vs. typically developing children rather than different age groups. Some developmental studies examined post-error slowing but the outcomes of these studies are inconsistent (for a review see Smulders et al., under review). Typically, in studies examining developmental change in post-error slowing, the results are interpreted in terms of conflict monitoring (Botvinick et al., 2001) and, based on event-related brain potentials or bold responses associated with error processing, it is usually suggested that conflict monitoring is still immature in children and, in some studies, not fully developed even in adolescents (e.g., Velanova et al., 2008; Braet et al., 2009). Obviously, the current pattern of findings is difficult to reconcile with the notion of developmental improvements in conflict monitoring, at least across the age span under study. By contrast, the current pattern suggests that the mechanisms engaged on stop-signal trials and resulting in response slowing on subsequent trials are already in place and mature in children as young as 7 years of age. A major difference between studies examining developmental change in post-error slowing and the current study focusing on performance adjustments following stop-signal trials is that errors are typically less frequent than stop-signals. It would be of considerable interest to examine developmental change in post-error and stop-signal slowing by calibrating the frequencies of error and stop-signal occurrence.

The findings obtained for the elderly adults differed from the results of the other age groups in three respects. First, the elderly adults did not slow following successful inhibits on both stop-signal tasks. Second, on both stop-signal tasks, the slowing following failed inhibits was disproportionally larger than in young adults. Third, the slowing following invalid stop-signal trials was disproportionally larger than in young adults. The observation that the elderly adults slowed following failed inhibits but did not following successful inhibits presents a challenge for the orienting interpretation of conflict adjustment proposed by Notebaert et al. (2009). According to the orienting account performance adjustments following successful and failed inhibits should be the same because the probability of success and failure is identical (around $50 \%)$. In this regard, the current findings are inconsistent with the prediction derived from the orienting account. The current findings are problematic also with regard to the conflict monitoring hypothesis (Botvinick et al., 2001). Conflict arises on all three stop-signal trials and, thus, performance adjustments should be observed on all trials following a stop-signal trial. The current data of elderly adults do suggest that performance adjustment is triggered by conflict but only when this conflict is resolved by the execution of an overt response, either correct, as on trials following invalid stop-signal trials, or incorrect, as on trials following failed inhibits.

A unified account of the data could be provided by assuming age-related changes in the balance between proactive and reactive performance adjustments in the stop-signal tasks. Although participants were instructed not to delay their speed of responding, and in spite of the tracking algorithm that should discourage participants from slowing down, the speed of responding on the stop-signal tasks was considerably slower than on the standard choice task in all age groups. The delay in responding could be considered an instance of proactive control exercised by the participants in an attempt to increase their chances to withhold the response when a stop-signal instructed them to do so. Proactive control in the current study is manifested also by differential slowing in the two stop-signal tasks. Larger delays were observed on the GST compared to the SST, presumably because proactive control is guided by the probability of stopping rather than stop-signal occurrence, as stop-signals might turn out to be invalid in the SST. Proactive control in stop-signal performance has been demonstrated in previous studies, both at the behavioral (e.g., Verbruggen and Logan, 2009) and neurocognitive level (for a review see Aron, 2011). Importantly, the slowing associated with the insertion of stop-signals was most pronounced for the elderly adults. Most likely, the occurrence of stop-signals indicated a greater need for caution in the elderly participants relative to the other age groups and, thus, response slowing on go trials is disproportionally larger in the elderly adults. This interpretation is consistent with the typical observation that elderly adults favor accuracy over speed (e.g., Band and Kok, 2000; Starns and Ratcliff, 2010). If elderly adults exercised more proactive control compared to the younger age groups there might be less need for them to resort to reactive control when they are confronted with a stop-signal trial. The current absence of a delay in responding on go trials following successful inhibits might suggest that proactive control was sufficient in dealing with the conflict between stopping and going. Apparently, proactive control was not sufficient on failed inhibits, which could signal the need for more control on subsequent trials and this is reflected in slowing on go trials following failed inhibits. Finally, 
the slowing on go trials following invalid stop-signal trials may suggest that reactive control is elicited on those trials by the added requirement of re-initiating the response that was stopped first in reaction to the detection of the stop-signal. The current interpretation is similar to the goal-priority hypothesis proposed recently by Bissett and Logan (2011) to account for the dynamic interplay between pro- and reactive control in the stop-signal paradigm. The goal-priority hypothesis assumes that stop-signals indicate the need for more caution and thus participants reduce their speed of responding. This might happen following a stop-signal trial (reactive control) but also during the anticipation of a stop-signal trial (proactive control) when, for example, explicit cues signal the importance of stopping (e.g., Verbruggen and Logan, 2009). It should be of considerable interest to further examine lifespan changes in the dynamic balance between pro- and reactive control in the stop-signal trial by cueing the importance of stopping vs. response speed.

In conclusion, the current findings indicate that, with regard to global and selective stopping, development, and aging are not simply two sides of the same coin. Developmental change in global and selective stopping revealed different trajectories, with global stopping maturing more rapidly than selective stopping. The pattern of findings is inconsistent with a simple "last-in-first-out" hypothesis of lifespan changes in (neuro)cognition. Importantly, children slowed their speed of responding following stop-signal trials, but the current data revealed little difference between children and

\section{REFERENCES}

Aron, A. R. (2011). From reactive to proactive and selective control: developing a richer model for stopping inappropriate responses. Biol. Psychiatry 69, 55-68.

Aron, A. R., and Verbruggen, F. (2008). Stop the presses: dissociating a selective from a global mechanism for stopping. Psychol. Sci. 19, 1146-1153.

Band, G. P. H., and Kok, A. (2000). Age effects on response monitoring in a mental-rotation task. Biol. Psychol. 51, 201-221.

Band, G. P. H., van der Molen, M. W., and Logan, G. D. (2003). Horse-race model simulations of the stop-signal procedure. Acta Psychol. (Amst.) 112, 105-142.

Band, G. P. H., van der Molen, M. W., Overtoom, C. C., and Verbaten, M. N. (2000). The ability to activate and inhibit speeded responses: separate developmental trends. J. Exp. Child Psychol. 75, 263-290.

Bedard, A., Nichols, S., Barbosa, J. A., Schachar, R., Logan, G. D., and Tannock, R. (2002). The development of selective inhibitory control across the life span. Dev. Neuropsychol. 21, 93-111.

Bergerbest, D., Gabrieli, J. D. E., Whitfield-Gabrieli, S., Kim, H., Stebbins, G. T., Bennett, D. A., and Fleischman, D. A. (2009).

young adults in this regard. Consequently, the type of reactive control exercised in the stop-signal paradigm seems to mature very rapidly. This finding has important implications for developmental theories of cognitive control (e.g., Durston and Casey, 2006), in particular for developmental concepts of conflict monitoring (e.g., Braet et al., 2009). All age groups delayed the speed of responding when stop-signals were inserted in the trial series and the delay was interpreted in terms of proactive control aimed at enhancing stopping success and in terms of an adjustment of response thresholds (e.g., Bissett and Logan, 2011). The data pattern obtained for the elderly adults suggest that they shifted the balance between proactive and reactive cognitive control toward proactive control. Most likely this shift is related to their willingness to trade speed for accuracy. Further investigation of the factors influencing the balance between pro- and reactive control in development and cognitive aging is an important goal for future research.

\section{ACKNOWLEDGMENTS}

This study was supported by NWO grants to Maurits W. van der Molen and Wery P. M. van den Wildenberg (MaGW grant 400-03261 and VENI grant 451-06-012, respectively). The support of the Netherlands Institute for Advanced Study in the Humanities and Social Sciences is also gratefully acknowledged. We thank Bert van Beek for programming the computer tasks and Marcus Spaan and Bert Molenkamp for technical support.

white matter tracts using diffusion tensor tractography. Neuroimage 46 530-541.

Dempster, F. N. (1992). The rise and fall of the inhibitory mechanism: toward a unified theory of cognitive development and aging. Dev. Rev. 12, 45-75.

Durston, S., and Casey, B. J. (2006) What have we learned about cognitive development from neuroimaging? Neuropsychologia 44 2149-2157.

Fleischman, D. A. (2007). Repetition priming in aging and Alzheimer's disease: an integrative review and future directions. Cortex 43, 889-897.

Fujiyama, H., Tandonnet, C., and Summers, J. J. (2011). Age-related differences in corticospinal excitability during a go/nogo task. Psychophysiology 48, 1448-1455.

Gamboz, N., Borella, E., and Brandimonte, M. A. (2009). The role of switching, inhibition, and working memory in older adults' performance in the Wisconsin card sorting test. Neuropsychol. Dev. Cogn. B Aging Neuropsychol. Cogn. 16, 260-284.

Gao, J., Wong-Lin, K., Holmes, P., Simen, P., and Cohen, J. D. (2009). Sequential effects in two-choice reaction time tasks: decomposition and synthesis of mechanisms. Neural Comput. 21, 2407-2436.
Huizinga, M., and van der Molen, M. W. (2010). Task switching and shifting between stopping and going: developmental change in betweentrial control adjustments. J. Exp. Child Psychol. 108, 484-503.

Johnstone, S. J., Dimoska, A., Smith, J. L., Barry, R. J., Pleffer, C. B., Chiswick, D., and Clarke, A. R. (2007). The development of stopsignal and go/nogo response inhibition in children aged 7-12 years: performance and event-related potential indices. Int. J. Psychophysiol. 63, 25-38.

Kail, R. (1997). The neural noise hypothesis: evidence from processing speed in adults with multiple sclerosis. Aging Neuropsychol. Cogn. 4, 157-165.

Kolev, V., Falkenstein, M., and Yordanova, J. (2005). Motor-response generation as a source of agingrelated behavioural slowing in choice-reaction tasks. Neurobiol. Aging 27, 1719-1730.

Kramer, A. F., Humphrey, D. G., Larish, J. F., Logan, G. D., and Strayer, D. L. (1994). Aging and inhibition: beyond a unitary view of inhibitory processing in attention. Psychol. Aging 9, 491-512.

Kray, J., Kipp, K. H., and Karbach, J. (2009). The development of selective inhibitory control: the influence of verbal labeling. Acta Psychol. (Amst.) 130, 48-57. 
Levitt, H. (1971). Transformed updown methods in psychoacoustics. J. Acoust. Soc. Am. 49, 467-477.

Li, X., Liang, Z., Kleiner, M., and Lu, Z. L. (2010). RTbox: a device for highly accurate response time measurements. Behav. Res. Methods 42, 212-225.

Logan, G. D. (1994). "On the ability to inhibit thought and action: a users' guide to the stop signal paradigm," in Inhibitory Processes in Attention, Memory and Language, eds D. Dagenbach and T. H. Carr (San Diego, CA: Academic Press), 189-239.

Logan, G. D., and Burkell, J. (1986). Dependence and independence in responding to double stimulation: a comparison of stop, change, and dual-task paradigms. J. Exp. Psychol. Hum. Percept. Perform. 12, 549-563.

Logan, G. D., and Cowan, W. B. (1984). On the ability to inhibit thought and action: a theory of an act of control. Psychol. Rev. 91, 295-327.

Luce, R. D. (1986). Response Times: Their Role in Inferring Elementary Mental Organization. New York, NY: Oxford University Press.

May, C. P., and Hasher, L. (1998). Synchrony effects in inhibitory control over thought and action. J. Exp. Psychol. Hum. Percept. Perform. 24, 363-379.

Mayda, A. B. V., Westphal, A., Carter, C. S., and DeCarli, C. (2011). Late life cognitive control deficits are accentuated by white matter disease burden. Brain 134, 1673-1683.

Meiran, N. (1996). Reconfiguration of processing mode prior to task performance. J. Exp. Psychol. Learn. Mem. Cogn. 22, 1423-1442.

Melis, A., Soetens, E., and van der Molen, M. W. (2002). Processspecific slowing with advancing age: evidence derived from the analysis of sequential effects. Brain Cogn. 49, 420-435.

Myerson, J., Hale, S., Wagstaff, D., Poon, L., and Smith, G. (1990). The information-loss model: a mathematical theory of age-related cognitive slowing. Psychol. Rev. 97, 475-487.

Notebaert, W., Houtman, F., Opstal, F. V., Gevers, W., Fias, W., and Verguts, T. (2009). Post-error slowing: an orienting account. Cognition 111, 275-279.

Oosterlaan, J., and Sergeant, J. A. (1996). Inhibition in ADHD, aggressive, and anxious children: a biologically based model of child psychopathology. J. Abnorm. Child. Psychol. 24, 19-36.

Ramautar, J. R., Kok, A., and Ridderinkhof, K. R. (2004). Effects of stop-signal probability in the stopsignal paradigm: the N2/P3 complex further validated. Brain Cogn. 56, 234-252.

Raven, J., Raven, J. C., and Court, J. H. (1993). Manual for Raven's Progressive Matrices and Mill Hill Vocabulary Scales. Oxford: Oxford Psychologists Press.

Raven, J. C., Court, J. H., and Raven, J. (1985). Manual for Raven's Progressive Matrices and Vocabulary Scales. Standard Progressive Matrices, Sect. 3. London: H. K. Lewis.

Ridderinkhof, K. R., Band, G. P. H., and Logan, G. D. (1999). A study of adaptive behavior: effects of age and irrelevant information on the ability to inhibit one's actions. Acta Psychol. (Amst.) 101, 315-337.

Rieger, M., and Gauggel, S. (1999). Inhibitory after-effects in the stop signal paradigm. Br. J. Psychol. 90, 509-518.

Rush, B. K., Barch, D. M., and Braver, T. S. (2006). Accounting for cognitive aging: context processing, inhibition or processing speed? Neuropsychol. Dev. Cogn. B Aging Neuropsychol. Cogn. 13, 588-610.

Smulders, S. F., Notebaert, W., Meijer, M., Crone, E. A., van der Molen, M. W., and Soetens, E. (2005). Sequential effects on speeded information processing: a developmental study. J. Exp. Child Psychol. 90, 208-234.

Somerville, L. H., and Casey, B. J. (2010). Developmental neurobiology of cognitive control and motivational systems. Neurobiology 20, 236-241.

Starns, J., and Ratcliff, R. (2010). The effects of aging on the speedaccuracy compromise: boundary optimality in the diffusion model. Psychol. Aging 25, 377-390.

Trewartha, K. M., Penhune, V. B., and Li, K. Z. (2011). Movement kinematics of prepotent response suppression in aging during conflict adaptation. J. Gerontol. B Psychol. Sci. Soc. Sci. 66, 185-194.

Vallesi, A. (2011). Targets and nontargets in the aging brain: a go/nogo event-related potential study. Neurosci. Lett. 487, 313-317.
Vallesi, A., McIntosh, A. R., and Stuss, D. T. (2011). Overrecruitment in the aging brain as a function of task demands: evidence for a compensatory view. J. Cogn. Neurosci. 23 , 801-815.

Vallesi,A., and Stuss, D. T. (2010). Excessive sub-threshold motor preparation for non-target stimuli in normal aging. Neuroimage 50, 1251-1257.

van Boxtel, G. J. M., van der Molen, M. W., Jennings, J. R. and Brunia, C. H. M. (2001). A psychophysiological analysis of inhibitory motor control in the stopsignal paradigm. Biol. Psychol. 58, 229-262.

van de Laar, M. C., van den Wildenberg, W. P. M., van Boxtel, G. J. M., and van der Molen, M. W. (2010). Processing of global and selective stop signals: application of Donders subtraction method to stop-signal task performance. Exp. Psychol. 57, 149-159.

van den Wildenberg, W. P. M., and van der Molen, M. W. (2003). The duration of response inhibition in the stop-signal paradigm varies with response force. Acta Psychol. (Amst.) $114,115-129$.

van den Wildenberg, W. P. M., and van der Molen, M. W. (2004a). Developmental trends in simple and selective inhibition of compatible and incompatible responses. J. Exp. Child. Psychol. 87, 201-220.

van den Wildenberg, W. P. M., and van der Molen, M. W. (2004b). Additive factors analysis of inhibitory processing in the stop-signal paradigm. Brain Cogn. 56, 253-266.

van der Schoot, M., Licht, R., Horsley, T. M., and Sergeant, J. A. (2005). Effects of stop signal modality, stop signal intensity and tracking method of inhibitory performance as determined by use of the stop signal paradigm. Scand. J. Psychol. 46, 331-341.

Velanova, K., Wheeler, M. E., and Luna, B. (2008). Maturational changes in anterior cingulate and frontoparietal recruitment support the development of error processing and inhibitory control. Cereb. Cortex 18 2505-2522.

Verbruggen, F., Liefooghe, B., and Vandierendonck, A. (2005). On the difference between response inhibition and negative priming: evidence from simple and selective stopping. Psychol. Res. 69, 262-271.
Verbruggen, F., and Logan, G. D. (2008). Response inhibition in the stopsignal paradigm. Trends Cogn. Sci. $12,418-424$

Verbruggen, F., and Logan, G. D. (2009). Proactive adjustments of response strategies in the stop-signal paradigm. J. Exp. Psychol. Hum. Percept. Perform. 35, 835-854.

Verbruggen, F., Logan, G. D., Liefooghe, B., and Vandierendonck, A. (2008). Short-term aftereffects of response inhibition: repetition priming or between-trial control adjustments? J. Exp. Psychol. Hum. Percept. Perform. 34, 413-426.

West, R., and Schwarb, H. (2006). The influence of aging and frontal function on the neural correlates of regulative and evaluative aspects of cognitive control. Neuropsychology 20, 468-481.

West, R. L. (1996). An application of prefrontal cortex function theory to cognitive aging. Psychol. Bull. 120, 272-292.

Williams, B. R., Ponesse, J. S., Schachar, R. J., Logan, G. D., and Tannock, R. (1999). Development of inhibitory control across the life span. Dev. Psychol. 35, 205-213.

Conflict of Interest Statement: The authors declare that the research was conducted in the absence of any commercial or financial relationships that could be construed as a potential conflict of interest.

Received: 01 September 2011; paper pending published: 04 October 2011; accepted: 12 November 2011; published online: 15 December 2011.

Citation: van de Laar MC, van den Wildenberg WPM, van Boxtel GJM and van der Molen MW (2011) Lifespan changes in global and selective stopping and performance adjustments. Front. Psychology 2:357. doi: 10.3389/fpsyg.2011.00357

This article was submitted to Frontiers in Cognition, a specialty of Frontiers in Psychology.

Copyright (c) 2011 van de Laar, van den Wildenberg, van Boxtel and van der Molen. This is an open-access article subject to a non-exclusive license between the authors and Frontiers Media SA, which permits use, distribution and reproduction in other forums, provided the original authors and source are credited and other Frontiers conditions are complied with. 